\title{
Triplex Doppler da artéria renal e a relação entre a ecobiometria dos rins com distância atlanto-coccígea e altura em Canis familiaris ${ }^{1}$
}

\author{
Expedito Jr Matos Santana², Poliana S. Beserra², Adriel B. Brito², Stefânia A. \\ Miranda $^{2}$, Elizabeth Nikolak ${ }^{2}$ e Sheyla F.S. Domingues ${ }^{2 *}$
}

\begin{abstract}
Santana E. Jr M., Beserra P.S., Brito A.B., Miranda S.A., Nikolak E. \& Domingues S.F.S. 2009. [Renal artery Triplex Doppler and the relationship between kidney ecobiometry with atlanto-coccyges distance (ACD) and height (H) in Canis familiaris.] Triplex Doppler da artéria renal e a relação entre a ecobiometria dos rins com distância atlanto-coccígea (DAC) e altura (H) em Canis familiaris. Pesquisa Veterinária Brasileira 29(10):809-815. Laboratório de Biologia e Medicina de Animais da Amazônia, Medicina Veterinária, Universidade Federal do Pará, Rua Hernane Lameira 556, Castanhal, PA 68743-050, Brazil. E-mail: shfarha@ufpa.br

The aim of this paper was to establish the relation between the kidney ecobiometry with atlanto-coccyges distance (ACD) and height $(\mathrm{H})$ in adult healthy dogs, to obtain normality parameters for assessment of renal size and volume, as well as establish reference values to evaluate kidney blood perfusion by the resistivity index $(\mathrm{RI})$ and pulsatility index $(\mathrm{PI})$ of main renal artery. The study was applied at 22 adult dogs, 11 males and 11 females. Previously, the DAC and $\mathrm{H}$ of all animals were measured. For ultrasonographic examination, the ultra-sound system HDI 4000 PHILIPS equipped with a multi-frequency microconvex transducer, Color Doppler and Spectral Doppler devices was used. The animals were placed into right or left lateral decubitus position, in agreement with the kidney to be assessed. The longitudinal (LD) and dorsoventral diameters (DVD) of kidney were measured in longitudinal plane, and the transverse diameter (TD) was determined in transversal section. The renal volume (V) was automatically calculated by the ultrasound software. With Triplex Doppler, the RI and PI of right and left main renal arteries were obtained. All data were represented in mean \pm SEM. Linear regression analyses were performed with renal LD, DVD, TD and V as dependent variable, and ACD and $\mathrm{H}$ as independent variable. $\mathrm{RI}$ and $\mathrm{PI}$ were compared between right and left renal arteries with Student's $t$-test. The LD, DVD, TD $(\mathrm{cm})$ and $\mathrm{V}(\mathrm{ml})$ mean measurements for the left and right kidneys were: $5.24 \pm 0.27$, $3.07 \pm 0.15,3.07 \pm 0.9,28.01 \pm 3.4$ and $4.50 \pm 0.19,2.88 \pm 0.14,2.71 \pm 0.15,21.27 \pm 2.6$. All regression analyses were significant for the intercept and regression coefficient $(P<0.01)$. There were statistical differences on $\mathrm{RI}$ and $\mathrm{PI}$ means between right and left renal arteries $(P=0.001)$. The $\mathrm{RI}$ and $\mathrm{PI}$ means of left and right renal artery obtained were: $0.62 \pm 0.08$ and $1.34 \pm 0.18 ; 0.70 \pm 0.06$ and $1.62 \pm 0.13$. The data obtained in the present paper can be used as parameters for evaluation of the renal size, volume and perfusion in adult dogs.
\end{abstract}

INDEX TERMS: Corporal conformation, ultrasound, renal perfusion, Dopplerfluxometry.

\footnotetext{
${ }^{1}$ Recebido em 12 de novembro de 2008.

Aceito para publicação em 27 de junho de 2009.

2 Laboratório de Biologia e Medicina de Animais da Amazônia, Medicina Veterinária, Universidade Federal do Pará (UFPA), Rua Hernane Lameira 556, Castanhal, PA 68743-050, Brasil. *Autor para correspondência: shfarha@ufpa.br
}

RESUMO.- O objetivo do trabalho foi estabelecer a relação entre a ecobiometria renal com medidas de conformação corporal como a distância atlanto-coccígea (DAC) e a altura $(\mathrm{H})$ de cães adultos saudáveis, obtendo-se parâmetros de normalidade para avaliar o tamanho e volume renal, bem como estabelecer valores de referência 
para avaliar a perfusão sanguínea dos rins por meio do índice de resistividade (IR) e do índice de pulsatilidade (IP) do ramo principal da artéria renal. No estudo foram utilizados 22 cães adultos sem raça definida, sendo 11 machos e 11 fêmeas. Os animais foram previamente aferidos quanto a DAC e a $\mathrm{H}$. Os exames ultra-sonográficos foram realizados com um aparelho HDI 4000 PHILIPS munido de um transdutor microconvexo multifreqüêncial (5-8 MHz), dispositivos Doppler Colorido e Doppler de Fluxo. Os animais foram posicionados em decúbito lateral direito ou esquerdo, de acordo com o rim a ser avaliado. Os diâmetros longitudinal (DL) e dorsoventral (DDV) dos rins foram mensurados na secção longitudinal e, o diâmetro transversal (DT) foi aferido no plano transversal. O volume $(\mathrm{V})$ foi calculado automaticamente pelo software do ultra-som. Com o uso do Triplex Doppler, o IR e o IP das artérias renais direita e esquerda foram obtidos. Todos os dados foram apresentados em média \pm EPM. Análises de regressão linear foram realizadas tendo o DL, DDV, DT e V como variáveis dependentes e a DAC e H como variáveis independentes. Os IR e IP dos rins direito e esquerdo foram comparados pelo teste $t$ de Student. A DAC variou de $54-78 \mathrm{~cm}$ para machos e $37-$ $71 \mathrm{~cm}$ para fêmeas e a altura variou entre $34-64 \mathrm{~cm}$ para os machos e $24-57 \mathrm{~cm}$ para as fêmeas. As médias obtidas para DL, DDV, DT e $\mathrm{V}$ dos rins esquerdo e direito foram: $5,24 \pm 0,27 \mathrm{~cm}, 3,07 \pm 0,15 \mathrm{~cm}, 3,07 \pm 0,9 \mathrm{~cm}$, $28,01 \pm 3,4 \mathrm{~mL}$ e $4,50 \pm 0,19 \mathrm{~cm}, 2,88 \pm 0,14 \mathrm{~cm}, 2,71 \pm 0,15 \mathrm{~cm}$, $21,27 \pm 2,6 \mathrm{~mL}$, respectivamente. As análises de regressão linear entre as medidas lineares e volume renal com a DAC e a $\mathrm{H}$ foram significativas para os interceptos e coeficientes de regressão $(P<0,01)$. Houve diferenças estatísticas quando comparado os IR e IP entre os rins direito e esquerdo $(P=0,001)$, sendo que as médias para IR e IP dos rins esquerdo e direito foram $0,62 \pm 0,08$; $1,34 \pm 0,18$ e $0,70 \pm 0,06 ; 1,62 \pm 0,13$; respectivamente. Os dados obtidos no presente trabalho podem auxiliar na avaliação do tamanho, volume e perfusão dos rins de cães adultos.

TERMOS DE INDEXAÇÃO: Conformação corporal, ultrassonografia, perfusão renal, dopplerfluxometria.

\section{INTRODUÇÃO}

Alterações no formato e tamanho dos rins comumente ocorrem como resultado de muitas enfermidades renais e a detecção destas mudanças são importantes para o diagnóstico e prognóstico das renopatias (Felkai et al. 1992). As mudanças anatômicas nos rins podem ser avaliadas pela radiografia (Finco et al. 1998), laparoscopia (Nyland \& Matoon 1995) e ultra-sonografia (Espada et al. 2006). O emprego da radiografia para estimar o volume renal em cães é efetivo apenas em $50 \%$ das abordagens, sendo necessária a administração de contrastes hiperosmóticos, o que resulta em diurese osmótica, alterando o tamanho renal durante urografia ou sendo ainda de pouca eficiência em cães com insuficiência renal
(Mareschal et al. 2007). A laparoscopia, por sua vez, é uma técnica invasiva de difícil execução na prática clínica veterinária diária, enquanto que a ultra-sonografia (US) vem ganhando espaço como exame de rotina por não ser invasivo e livre de efeitos colaterais ao operador e paciente (Domingues et al. 2007).

As imagens ultra-sonográficas bidimensionais (2D) em modo $B$ podem ser obtidas em tempo real, em qualquer secção e orientação espacial, o que permite detectar movimentos, analisar a biometria e avaliar patologias que comprometem a integridade morfológica e funcional dos órgãos (King 2006). Na US 2D em modo B do rim, é possível delimitar a cápsula, o córtex, a medula, o seio renal, a crista renal, as pirâmides, a pelve, bem como os ureteres proximais (Banks 1992), além de permitir identificar as diferenças de ecogenicidade do parênquima renal e suas alterações (Konde et al. 1984, Churchill et al. 1999, Gaschen et al. 2000).

No entanto, são escassas as informações consistentes a respeito do tamanho e volume renal normal em cães saudáveis. Estudos correlacionando as medidas lineares e o volume renal com o peso canino tem sucesso limitado (Felkai et al. 1992, Sampaio \& Araújo 2002, Nyland et al. 2005), uma vez que o tamanho do órgão pode ser superestimado em um cão adulto subnutrido, assim como subestimado em cães adultos obesos. Recentemente, temse buscado correlacionar o diâmetro longitudinal do rim com o diâmetro da artéria aorta abdominal em cães clinicamente normais, com intuito de estabelecer parâmetro de normalidade para o tamanho renal (Mareschal et al. 2007). No entanto, durante um exame ultra-sonográfico transabdominal 2D em modo $B$, nem sempre é possível mensurar com precisão o diâmetro da artéria aorta, o que pode dificultar a utilização do mesmo como parâmetro de correlação com a biometria renal durante a prática clínica. Dessa forma, estudos relacionando o tamanho e o volume do rim normal de cães, com medidas de conformação corporal fáceis de serem obtidas e reproduzidas são necessários, como por exemplo, a distância atlanto-coccígea e a altura.

Outro método importante oferecido pela US são os modos Doppler Colorido e Doppler de Fluxo (Triplex Doppler) que podem ser utilizados para identificar a irrigação sanguínea renal, com informações em relação à presença, o direcionamento e o tipo de fluxo sanguíneo (Szatmári et al. 2001). O Triplex Doppler possibilita executar avaliações quantitativas e qualitativas do fluxo sanguíneo (Domingues et al. 2007). Informações quantitativas produzidas pelo Triplex Doppler incluem as velocidades média, máxima e mínima do fluxo sanguíneo. A partir dos valores dessas velocidades, o índice de resistividade (IR) (Pourcelot 1974) e o índice de pulsatilidade (IP) (Gosling et al. 1974) são calculados, sendo que a vantagem do uso desses índices é que os seus valores independem do ângulo de insonação (Domingues et al. 2007). A elevação dos valores do IR e IP da artéria renal representam à diminuição do fluxo sanguíneo do rim, o que demonstra um possível processo patológico, tais como nefropatias 
obstrutivas, obstrução renal aguda, necrose tubular aguda ou rejeição alográfica aguda (Gaschen et al. 2000). Entretanto, para o diagnóstico de doença vascular renal é necessário o conhecimento dos IR e IP normais da artéria renal. Em cães ainda são poucos os trabalhos visando o estudo em Triplex Doppler das artérias renais.

O objetivo dos autores foi relacionar o diâmetro longitudinal (DL), transversal (DT), dorsoventral (DDV) e volume (V) do rim de cães adultos saudáveis mensurados por meio da US em modo $B$, com medidas de conformação corporal canino como distância atlanto-coccígea (DAC) e a altura $(\mathrm{H})$, bem como descrever os valores normais dos IR e IP para as artérias renais direita e esquerda através do Triplex Doppler. Os dados obtidos no presente estudo podem ser importantes para auxiliar no reconhecimento e avaliação de nefropatias, que cursam com o aumento (hidronefrose) ou diminuição (estenose da artéria renal) do tamanho dos rins.

\section{MATERIAL E MÉTODOS}

Animais experimentais. No estudo foram utilizados 22 cães adultos sem raça definida (SRD), sendo 11 fêmeas, com 2-10 anos de idade e pesando 4-27 kg, e 11 machos com 2-8 anos de idade e pesando $9-40 \mathrm{~kg}$. Todos os cães utilizados neste experimento eram alimentados com ração canina comercial, viviam em um ambiente que favorecia o bem-estar animal (banho, alimento, espaço, higiene ambiental); eram imunizados por vacinas comerciais e saudáveis ao exame físico e clínico. Os exames ultra-sonográficos foram realizados no Laboratório de Biologia e Medicina de Animais da Amazônia (BIOMEDAM, UFPA) da Universidade Federal do Pará.

Biometria corpórea. Os animais foram previamente aferidos com o auxílio de uma fita métrica quanto a distância da articulação atlanto-occipital, a partir da crista da nuca, até a primeira vértebra coccígea (DAC). A altura $(\mathrm{H})$ foi mensurada da superfície palmar à cernelha.

Exame ultra-sonográfico. Previamente ao exame ultrasonográfico, foi realizada uma tricotomia na região lateral do abdômen, caudal a última costela esquerda, e no lado direito a partir do $11^{\circ}$ espaço intercostal (Nyland et al. 2005), estendendo-se $1,5 \mathrm{~cm}$ caudalmente à $13^{\mathrm{a}}$ costela (Gaschen et al. 2000). O mesmo procedimento foi realizado na região ventral do abdômen para facilitar a visualização ultrasonográfica da bexiga (Cruz-Arambulo \& Wrigley 2003). Os exames ultra-sonográficos em modo $B$ foram realizados com um aparelho PHILIPS HDI 4000 (Philips Medical Systems, Bothel, WA, EUA) munido de um transdutor microconvexo multifreqüêncial (5-8 MHz) e dispositivos Doppler Colorido e Doppler de Fluxo. Os animais foram posicionados em decúbito lateral direito ou esquerdo, de acordo com o rim a ser avaliado. No momento do exame, foi utilizado gel de transmissão hidrossolúvel para US (Aquasonic, Parker Laboratories, Fairfield, New Jersey 07004), como meio de contato entre o transdutor e a pele, com o intuito de eliminar a interface de ar entre a sonda e a região de interesse. Os diâmetros longitudinais (DL) e dorsoventrais (DDV) dos rins foram mensurados em uma secção sagital e, o diâmetro transversal (DT) em um plano transversal. O volume (V) renal foi calculado automaticamente pelo software do US a partir das medidas lineares, segundo a fórmula para o volume de um elipsóide.
Com o auxílio do dispositivo Doppler Colorido, a artéria renal foi localizada, sendo em seguida, o modo Doppler de Fluxo acionado para a obtenção do amostral pelo Doppler pulsátil, o calipter foi posicionado no centro da artéria renal. Foram consideradas para os cálculos dos IR e IP pelo menos três ondas de fluxo nítidas e consecutivas, calculadas automaticamente pelo equipamento de ultra-som a partir das seguintes fórmulas:

\section{$\underline{I R}=$ Pico de velocidade sistólica - Velocidade diastólica final Pico velocidade sistólica \\ IP = Pico de velocidade sistólica - Velocidade diastólica final Velocidade média do ciclo}

Análise estatística. Todos os dados obtidos estão apresentados em média \pm erro padrão. As medidas lineares e volumes dos rins direito e esquerdo foram comparados pelo teste $t$ de Student. As análises de regressão simples foram realizadas tendo os DL, DDV, DT e V como variáveis dependentes, e a distância atlanto-coccígea (DAC) e a altura (H) dos animais como variáveis independentes. Os IR e IP dos rins direito e esquerdo foram comparados pelo teste $t$ de Student. Todos os testes foram aplicados a $1 \%$ de probabilidade. As análises foram realizadas com o auxilio do programa estatístico Stat View (SAS Institute Inc., Cary, NC, USA).

\section{RESULTADOS}

As médias da DAC e $\mathrm{H}$ dos animais foram de $57,9 \pm 2,5 \mathrm{~cm}$ $(37-80 \mathrm{~cm})$ e $41,72 \pm 2,5(23-64 \mathrm{~cm})$, respectivamente. Os rins apresentaram córtex (Fig.1A), medula e a pelve renal (Fig.1B) dentro do limite da normalidade, quando comparada à ecogenicidade do parênquima hepático.

A utilização da sonda microconvexa multifrequêncial possibilitou a aquisição das mensurações lineares dos rins direito e esquerdo (Fig.2A,B), bem como as análises das artérias renais por meio do Triplex Doppler.

O Quadro 1 apresenta as médias \pm erro padrão das medidas lineares e volume dos rins. Houve diferenças estatísticas entre os DL, DDV, DT e V quando comparados os rins direito e esquerdo. Nos Quadros 2 e 3 estão representadas as análises de regressão entre as medi-

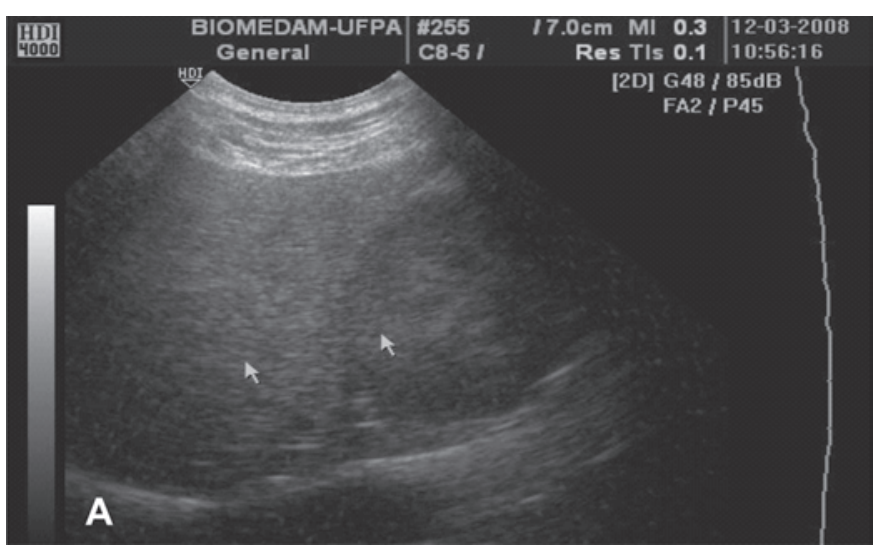

Fig.1. (A) Vista transversal do rim direito, notar a presença do fígado isoecóico em relação ao rim: córtex renal e parênquima hepático (setas). 


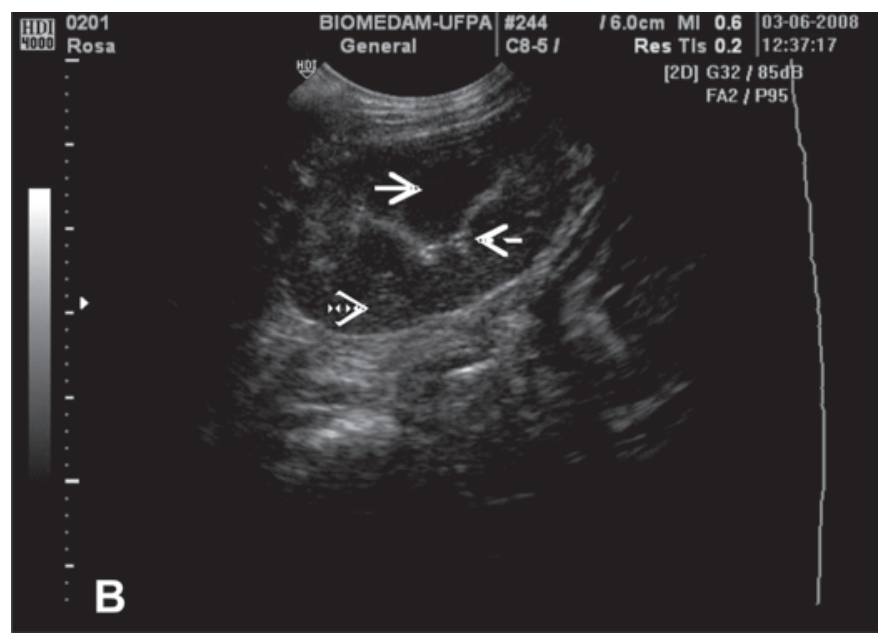

Fig.1. (B) Observar a delimitação da região de córtex (seta branca pontilhada), medular (seta branca continua), e pelve renal (seta branca tracejada).

das ecobiométricas renais com a DAC e a $\mathrm{H}$ dos animais, sendo que todas foram significativas para os interceptos e coeficientes de regressão $(P<0.01)$.

Todos os animais utilizados apresentavam a vesícula urinária ultra-sonograficamente normal, sem presença de sedimento e pontos ecóicos flutuantes em seu conteúdo,
Quadro 1. Médias \pm erro padrão dos diâmetros longitudinal (DL), dorsoventral (DDV), transversal (DT) e volume (V) dos rins direito e esquerdo

\begin{tabular}{lcc}
\hline & Rim direito & Rim esquerdo \\
\hline Longitudinal $(\mathrm{cm})$ & $4,29 \pm 0,19^{\mathrm{a}}$ & $5,24 \pm 0,25^{\mathrm{b}}$ \\
Dorsoventral $(\mathrm{cm})$ & $2,75 \pm 0,14^{\mathrm{a}}$ & $2,96 \pm 0,16^{\mathrm{b}}$ \\
Transversal $(\mathrm{cm})$ & $2,72 \pm 0,14^{\mathrm{a}}$ & $2,95 \pm 0,15^{\mathrm{b}}$ \\
Volume $(\mathrm{mL})$ & $19,14 \pm 2,57^{\mathrm{a}}$ & $27,67 \pm 3,5^{\mathrm{b}}$
\end{tabular}

a-b Diferentes letras indicam diferenças estatística entre rim direito e esquerdo $(P<0,01)$.

com contornos interno e externo regulares, onde a parede foi visualizada como duas linhas hiperecóicas finas separadas ao meio por uma fina linha hipoecóica (Fig.3).

Em 11 cães foi possível identificar as artérias renais direita e esquerda pelo Doppler Colorido (Fig.4A) e obter o cálculo por Doppler de Fluxo dos IR e IP (Fig.4B), sem a necessidade de administrar sedativos aos animais. $O$ transdutor microconvexo multifreqüencial $(5-8 \mathrm{MHz})$ é preciso na recaptação das ondas, o que facilita a coleta de dados. As médias \pm erro padrão dos IR e IP para o rim direito e esquerdo foram $0,71 \pm 0,02$ e 1,56 $\pm 0,04 ; 0,63 \pm 0,02$

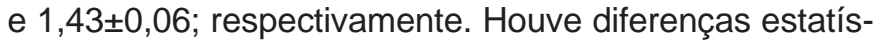
ticas quando comparados o IR e o IP entre as artérias renais direita e esquerda $(P=0,001)$.
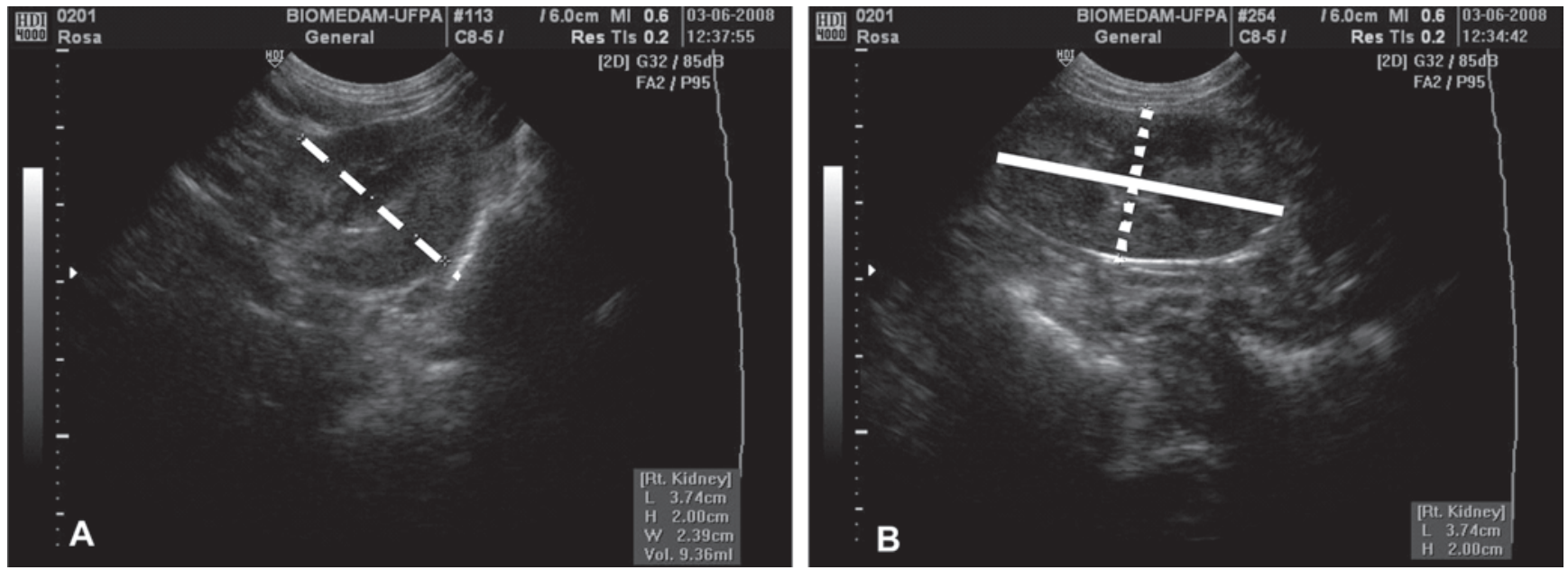

Fig.2. Ecobiometria renal: (A) Plano transversal para mensuração do Diâmetro Transversal, DT (linha branca tracejada). (B) Plano longitudinal para mensuração do Diâmetro Longitudinal, DL (linha branca contínua) e Diâmetro Dorsoventral, DDV (linha branca pontilhada). O volume $(\mathrm{V})$ é calculado automaticamente pelo software do ultra-som a partir das três primeiras medidas.

Quadro 2. Análise de regressão das medidas biométricas dos rins direito e esquerdo em relação à distância atlanto-coccígea (DAC) de Canis familiaris SRD adultos

\begin{tabular}{lccccc}
\hline & \multicolumn{2}{c}{ Rim direito } & & Rim esquerdo & \\
\cline { 2 - 3 } \cline { 5 - 6 } & Equação & $R^{2}$ & & Equação & $\mathrm{R}^{2}$ \\
\hline Longitudinal (DL) & $\mathrm{DL}=0,75+0,06 \mathrm{DAC}$ & 0,82 & & $\mathrm{DL}=-0,64+0,1 \mathrm{DAC}$ & 0,96 \\
Dorsoventral (DDV) & $\mathrm{DDV}=-0,35+0,05 \mathrm{DAC}$ & 0,97 & & $\mathrm{DDV}=-0,3+0,06 \mathrm{DAC}$ & 0,92 \\
Transversal (DT) & $\mathrm{DT}=-0,19+0,05 \mathrm{DAC}$ & 0,97 & & $\mathrm{DT}=-0,26+0,05 \mathrm{DAC}$ & 0,97 \\
Volume (V) & $\mathrm{V}=-27+0,8 \mathrm{DAC}$ & 0,88 & & $\mathrm{~V}=-24,5+0,8 \mathrm{DAC}$ & 0,88
\end{tabular}

$\mathrm{R}^{2}=$ coeficiente de determinação. 
Quadro 3. Análise de regressão das medidas biométricas dos rins direito e esquerdo em relação à altura $(\mathrm{H})$ de Canis familiaris SRD adultos

\begin{tabular}{|c|c|c|c|c|}
\hline & \multicolumn{2}{|c|}{ Rim direito } & \multicolumn{2}{|l|}{ Rim esquerdo } \\
\hline & Equação & $R^{2}$ & Equação & $R^{2}$ \\
\hline Longitudinal (DL) & $\mathrm{DL}=1,85+0,05 \mathrm{H}$ & 0,90 & $\mathrm{DL}=1,96+0,07 \mathrm{H}$ & 0,89 \\
\hline Dorsoventral (DDV) & $\mathrm{DDV}=0,85+0,04 \mathrm{H}$ & 0,90 & $\mathrm{DDV}=1,05+0,04 \mathrm{H}$ & 0,89 \\
\hline Transversal (DT) & $\mathrm{DT}=0,95+0,04 \mathrm{H}$ & 0,88 & $\mathrm{DT}=1,35+0,03 \mathrm{H}$ & 0,81 \\
\hline Volume (V) & $V=-7,83+0,6 H$ & 0,85 & $V=-17,51+1,07 \mathrm{H}$ & 0,91 \\
\hline
\end{tabular}

$\overline{\mathrm{R}^{2}=\text { coeficiente de determinação. }}$.

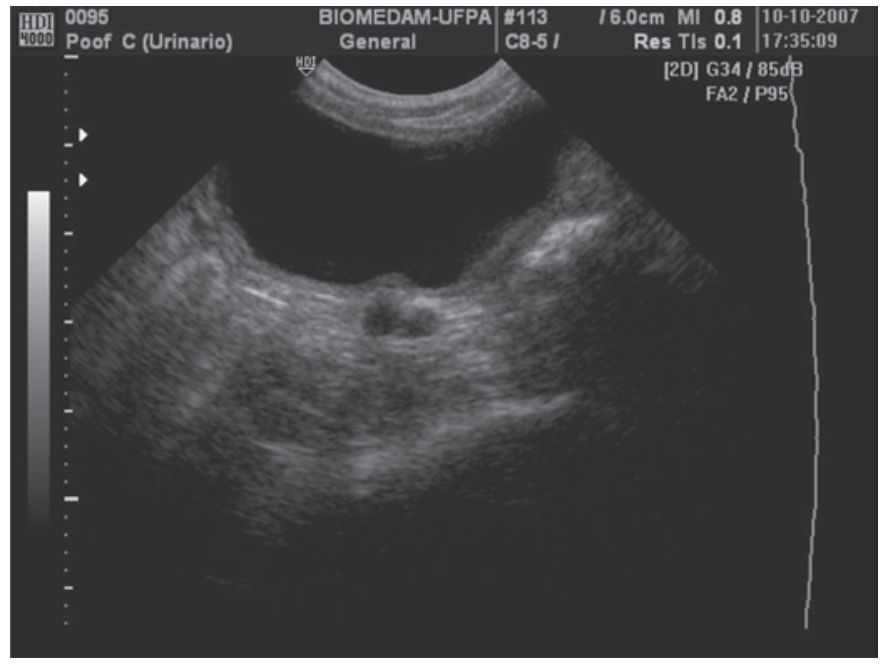

Fig.3. Vista transversal da bexiga que normalmente possui conteúdo anecóico, sem presença de sedimento, ausência de espessamento de mucosa, e com bordas regulares. Notar a parede como duas linhas hiperecóicas finas separadas ao meio por uma fina linha hipoecóica.

\section{DISCUSSÃO}

Os rins dos cães utilizados neste experimento apresentavam ecogenicidade normal no córtex, medula e pelve, como os achados descritos por Konde (1984) e Gaschen (2000). Morfologicamente, o rim direito foi menor em relação ao esquerdo nas medidas lineares e volume. Tem sido descrito que a localização do rim direito é dificultada pela penúltima e ultima costela direita, bem como devido à compressão exercida pelo lobo caudado do fígado e alças intestinais repletas de gases (Nyland et al. 2005). Diante destas situações, a visualização ultrasonográfica do rim direito pode ser prejudicada. No entanto, a utilização da sonda microconvexa no presente trabalho contornou as dificuldades de visualização ecográfica do rim direito, por exigir uma pequena janela acústica, facilitando a aquisição de imagens entre os espaços intercostais (1 $11^{\circ}$ ao $\left.13^{\circ}\right)$, diferentemente das sondas lineares e convexas (King 2006), que possuem uma janela acústica grande e não são eficientes para aquisição de imagens entre os espaços intercostais, resultando na formação de sombras acústicas. Sampaio \& Araújo (2002) e Nyland et al. (1989) possuem trabalhos onde as medidas do rim direito são semelhantes ao esquerdo por mensurações ultra-sonográficas, no entanto esses autores utili-

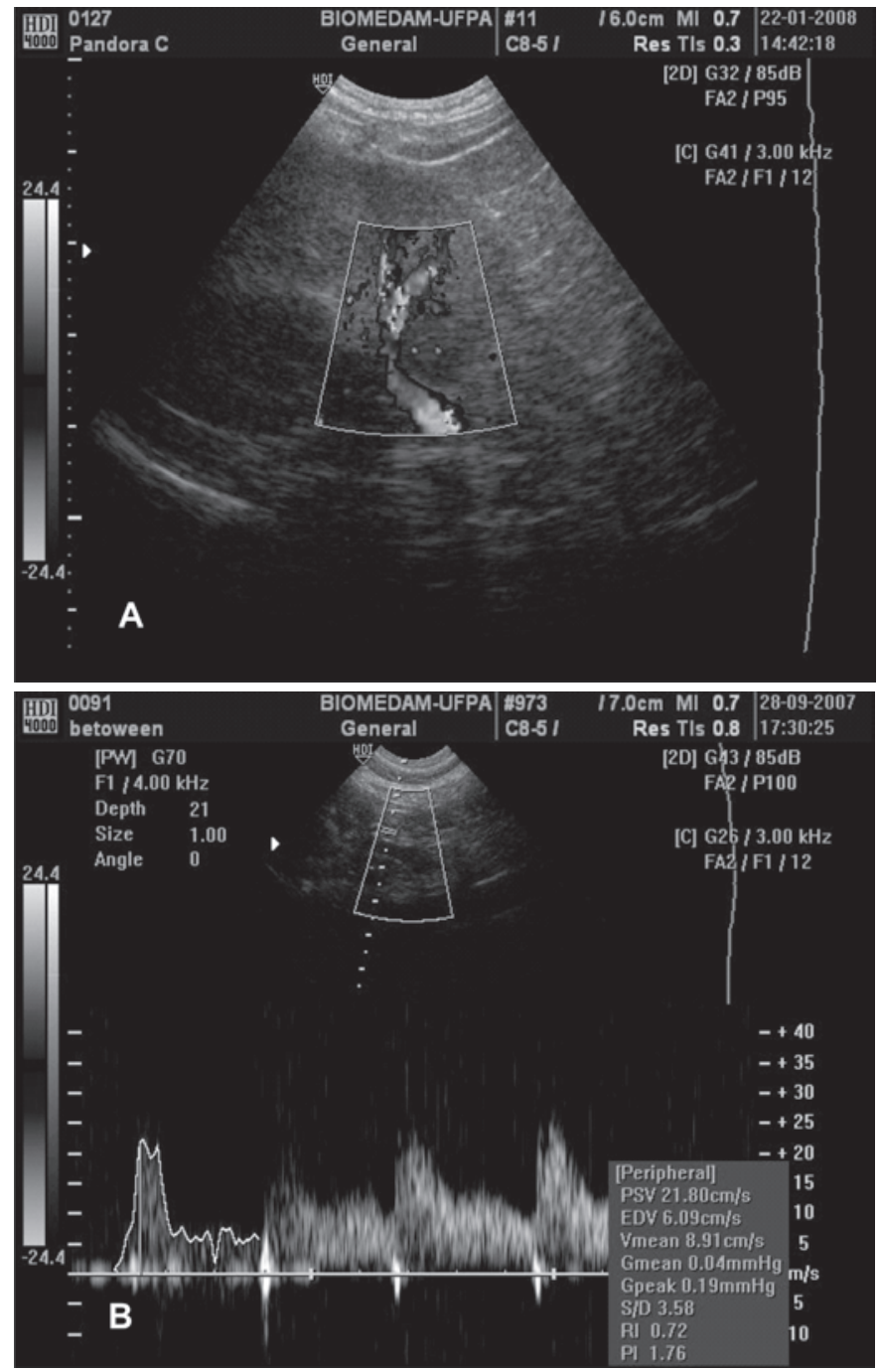

Fig.4. (A) método Doppler Colorido para localizar a artéria renal na região de hilo. (B) Triplex Doppler para calcular os valores de índice de resistividade (IR) e índice de pulsatilidade (IP).

zaram sondas lineares e convexas para a avaliação de ambos os rins.

A relação entre DL, DDV, DT e $V$ com as medidas de conformação corporal, como DAC e H, são parâmetros confiáveis para auxiliar o diagnóstico de enfermidades renais em cães adultos, considerando que as análises de regressão foram significativas tanto para as medidas lineares quanto para os volumes renais, principalmente para 
cães de meia idade, pois alguns estudos relatam sobre uma possível alteração no tamanho e volume renal em decorrência de uma alimentação rica em proteína, por um período prolongado, em cães geriatras (Churchill et al. 1999). Aproximadamente $80 \%$ dos pacientes adultos podem sofrer de hipertrofia renal compensatória, que tem como causa a redução da massa do rim contralateral e/ ou aumento da taxa de filtração glomerular, sendo que 10 a 15\% nas mudanças dos parâmetros lineares renais são por hipertrofia compensatória em cães geriatras (Emamian et al. 1993, Churchill et al. 1999).

As mensurações dos DL, DDV e DT são importantes para calcular o volume renal, que em muitas situações aumenta sobremaneira em relação às medidas corpórea do cão, como em caso de rejeição pós-transplante (Pollard 1999). Para calcular o $V$ com as fórmulas de regressão linear descritas no presente trabalho, os animais terão que apresentar H mínima de $23 \mathrm{~cm}$ e/ou DAC mínimo de $37 \mathrm{~cm}$. Entretanto, com os cálculos dos DL, DDV e DT, por meio das fórmulas demonstradas nos Quadros 2 e 3, é possível estimar o $\mathrm{V}$ teórico normal a partir do modelo de um elipsóide como proposto por Barr (1990), segundo a fórmula: $V=D L \times D D V \times D T \times 0,523$. Com o auxílio do Doppler Colorido, detectamos o ramo principal da artéria renal, tanto em plano transversal quanto no sagital, o que facilita o posicionamento do calípter para executar a dopplerfluxometria e obter os valores dos IR e IP. O rim é um órgão que tem o seu posicionamento influenciado pelos os movimentos respiratórios, sendo sincrônico com os mesmos, portanto o estresse, exercícios, digestão e orientação gravitacional podem dificultar a coleta dos dados no momento do exame. Dessa forma, para se ter eficiência na utilização do Doppler de Fluxo é imprescindível à colaboração do paciente, muitas vezes obtida somente por contenção química. No entanto, no presente trabalho, a utilização da sonda microconvexa facilitou a obtenção dos IR e IP, devido sua pequena dimensão quando comparada com outras sondas, sendo ideal para executar manobras em espaços de difícil acesso e, por ser menos incômoda ao animal no momento da realização do exame ultra-sonográfico.

Os cães utilizados nesse experimento não foram submetidos à sedação, pois este procedimento pode alterar os valores normais dos IR e IP (Rivers et al. 1997, Melo et al. 2006). Melo et al. (2006) relataram um valor de 0,70 para o IR do ramo principal da artéria renal em cães adultos SRD, sendo esse próximo ao proposto por Zubarev (2001) como normal para humanos. No entanto, Melo et al. (2006) não descreveram o valor do IP para o ramo principal da artéria renal em Canis familiaris, sendo este um parâmetro importante para avaliar a perfusão do órgão durante o ciclo cardíaco completo.

No presente trabalho, demonstrou-se que os valores de IR e IP do rim esquerdo foram menores que os do rim direito, indicando que a perfusão é maior no primeiro. Este fato pode estar relacionado à necessidade de uma maior perfusão no rim esquerdo, visto que este apresentou me- didas ecobiométricas lineares e volume maiores quando comparado com o rim direito.

\section{CONCLUSÕES}

De acordo com o presente trabalho, a relação entre a DAC e a $\mathrm{H}$ com as medidas ecobiométricas renais foram significativas, demonstrando que o tamanho do rim é influenciado pela conformação corporal do cão.

As análises de regressão linear obtidas entre as medidas lineares e volume dos rins com a DAC e a $\mathrm{H}$ podem auxiliar na avaliação do paciente, diagnosticando se este sofre ou não de alterações do tamanho e volume renais.

Com a utilização da sonda microconvexa foi possível obter informações de dopplerfluxometria do ramo principal das artérias renais direita e esquerda, bem como mensurar a perfusão sangüínea do órgão por meio dos valores de IR e IP, demonstrando que o Triplex Doppler pode ser utilizado como técnica de rotina clínica para diagnosticar e monitorar enfermidades que cursam com distúrbios circulatórios renais.

\section{REFERÊNCIAS}

Banks W.J. 1992. Sistema urinário, p.481-498. In: Ibid. (Ed.), Histologia Veterinária Aplicada. 2ª ed. Editora Manole, São Paulo.

Barr F.J. 1990. Evaluation of ultrasound as a method of assessing renal size in the dog. J. Small Anim. Pract. 31:174-179.

Churchill J.A., Feeney D.A., Fletcher T.F., Osborne C.A. \& Polzin D.J. 1999. Effects of diet and aging on renal measurements in uninephrectomized geriatric bitches. Vet. Radiol. Ultrasound. 40:233-240.

Cruz-Arambulo R. \& Wrigley R. 2003. Ultrasonography of the acute abdômen. Clin. Tech. Small Anim. Pract. 18:20-21.

Domingues S.F.S., Caldasbussiere M., Martins N. \& Carvalho R. 2007. Ultrasonographic imaging of the reproductive tract and surgical recovery of oocytes in Cebus apella (capuchin monkeys). Theriogenology 68:1251-1259.

Emamian S.A., Nielsen M.B., Pedersen J.F. \& Ytte L. 1993. Kidney dimensions at sonography: Correlation with age, sex, and habitus in 665 adult volunteers. Am. J. Roentgenol. 160:83-86.

Espada Y., Novellas R. \& Gopegui R.R. 2006. Renal ultrasound in dogs and cats. Vet. Res. Commun., 30(Suppl.1):133-137.

Felkai C.S., Vorös K. \& Vrabely T. 1992. Ultrasonographic determination of renal volume in the dog. Vet. Radiol. Ultrasound. 33:292-296.

Finco D.R., Stiles N.S., Kneller S.K., Lewis R.E. \& Barrett R.B. 1998. Radiologic estimation of kidney size of the Dog. J. Am. Vet. Med. Assoc. 159:995-1002.

Gaschen L., Menninger K. \& Schuurman H.J. 2000. Ultrasonography of the normal kidney in the Cynomolgus monkey (Macaca fascicularis): morphologic and Doppler findings. J. Med. Primatol., Munksgaard, 29:76-84.

Gosling R.G., Dunbar G., King D.H., Newman D.L., Side C.D., Woodcock J.P., Fitzgerald D.E., Keates J.S. \& MacMillan D. 1974. The quantitative analysis of occlusive peripheral arterial disease by a non-intrusive ultrasonic technique. Angiology 22:52-55.

King A.M. 2006. Development, advances and applications of diagnostic ultrasound in animals. Vet. J. 171:408-420.

Konde L.J., Wrigley R.H., Park R.D. \& Lebel J.L. 1984. Ultrasonographic anatomy of the normal canine kidney. Vet. Radiol. 25:173-178.

Mareschal A., D’Anjou M., Moreau M., Alexander K. \& Beauregard G. 2007. Ultrasonographic measurement of kidney-to-Aorta ratio as a method of estimating renal size in dogs. Vet. Radiol. Ultrasound. 48:434-438. 
Melo M.B., Veado J.C.C., Silva E.F., Moreira S.M. \& Passos L.M.F. 2006. Dopplerfluxometria das Artérias Renais: valores normais das velocidades sistólica e diastólica e do índice resistivo nas artérias renais principais. Arq. Bras. Med. Vet. Zootec. 58-4:691-693.

Nyland T.G. \& Matoon J.S. 1995. Ultrasonography of the urinary tract and adrenal glands, p.95-124. In: Ibid. (Eds), Veterinary Diagnostic Ultrasound. W.B. Saunders, Philadelphia.

Nyland T.G., Kantrowitz B.M., Fisher P., Olander H.J. \& Hornhof W.J. 1989. Ultrasonic determination of kidney volume in the dog. Vet. Radiol. 30:174-180.

Nyland T.G., Matoon J.S., Herrgesell E.J. \& Wisner. E.R. 2005. Trato urinário, p.161-194. In: Ibid. (Eds), Ultra-som Diagnóstico em Pequenos Animais. $2^{\mathrm{a}}$ ed. Roca, São Paulo.

Pollard R., Nyland T.G., Bernsteen L., Gregory C.R. \& Hornof W.J. 1999. Ultrasonographic evaluation of renal autografts in normal cats. Vet. Radiol. Ultrasound. 40:380-385.
Pourcelot L. 1974. Applications cliniques de l'examen Doppler transcutane, p.213-240. In: Peronneau P. (Ed.), Velocimetrie Ultrasonore Doppler. Semin Inserm, Paris.

Rivers B.J., Walter P.A., Letourneau J.G., Finlay D.E., Ritenour E.R., King V.L., O'Brien T.D. \& Polzin D.J. 1997. Duplex Doppler estimation of resistive index in arcuate arteries of sedated, normal female dogs: implications for use in the diagnosis of renal failure. J. Am. Anim. Hosp. Assoc., 33:69-76.

Sampaio K.M.O.R. \& Araújo R.B. 2002. Ultra-sonografia de características lineares e estimativas do volume de rins de cães. Arq. Bras. Med. Vet. Zootec. 54(3):248-254.

Szatmári V., Sótonyi P. \& Vörös K. 2001. Normal duplex Doppler waveforms of the major abdominal blood vessels in dogs: A review. Vet. Radiol. Ultrasound 42:93-107.

Zubarev A.V. 2001. Ultrasound of renal vessels. Eur. Radiol. 11:19021915. 